\title{
Left Ventricular Hypertrabeculation / Noncompaction Associated with a SDHD Mutation
}

\author{
Josef Finsterer $^{1 *}$ and Sinda Zarrouk-Mahjoub ${ }^{2}$ \\ ${ }^{1}$ Krankenanstalt Rudolf stiftung, Austria, Europe \\ ${ }^{2}$ Genomics Platform, Pasteur Institute of Tunis, Tunisia
}

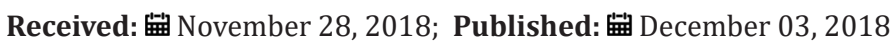

*Corresponding author: Josef Finsterer, Krankenanstalt Rudolf stiftung, Postfach 20, 1180 Vienna, Austria, Europe

\section{Letter to the Editor}

In a recent article Alston et al. reported about a male neonate with mitochondrial disorder (MID) due to the novel homozygous mutation c. $275 \mathrm{~A}>\mathrm{G}$ in the $S D H D$ gene [1]. The only affected organ in this boy was the heart [1]. We have the following comments and concerns. In the vast majority of the cases noncompaction, also known as left ventricular hypertrabeculation (LVHT), is congenital [2] and LVHT can be detected already prenatally by fetal echocardiography [3]. Which was the reason why LVHT was recognised neither on fetal echocardiography nor on fetal cardiac MRI and not before postnatal echocardiography? Since it is quite unusual that LVHT is missed on echocardiography and cardiac MRI it can be speculated that it developed after the last cardiac imaging at gestation week 32. Were fetal echocardiographies and fetal cardiac MRI reviewed after detection of LVHT on postnatal echocardiography and autopsy?

Reasons for missing LVHT on echocardiography could be that the investigating examiner is not familiar with the entity, that he does not depict the apex carefully, that the left ventricle is severely dilated, that there is marked thickening of the left ventricular myocardium, as in the presented patient, or that there are abnormalities mimicking LVHT, such as intraventricular thrombus formation, apical type of left ventricular hypertrophy, myocardial calcification, aberrant bands, or intraventricular abscess [4]. LVHT may not only be associated with mutations in mtDNA genes, nuclear genes encoding for the mitochondrial proteome or the TAZ gene, but also in the dystrophin, DMPK, $\alpha$-DTNA, RYR1, ITGA7, MYH7B, LAMP2, GAA, GBEI, MADD, COL7A1, MMACHC, PMP22, FXN, ß-globin, PLEC1, GLA, NKX2-5, MYH7, LDB3/ZASP, ACTC1, TNNT2, MYBPC3, TPM1, TNNI3, LMNA, and SCN5A genes [5]. Though LVHT is most frequently associated with mutations in mitochondrial genes [6], it cannot be excluded that the described patient also carried a second mutation in one of the many other genes associated with LVHT.

Since LVHT occurs familiarly, it would be interesting to know if the parents or other relatives of the boy were investigated for LVHT and if LVHT was found in any other family member? Of particular interest is if relatives who also carried the SDHD mutation also presented with LVHT. Simultaneous occurrence of the mutation and LVHT would be a strong argument for a causal relation. Arguments against a causal relation, however, are that LVHT is associated with mutations in a huge number of different genes, that it occurs only in a small number of mutation carriers, and that family members of a patient with LVHT often present with cardiac abnormalities other than LVHT, such as hypertrophic cardiomyopathy, dilative cardiomyopathy, or arrhythmias. Overall, this interesting case could be further up-valued by clarifying if LVHT was already present prenatally, which other family members were carrying the mutation and had LVHT, if organs other than the heart were affected, and if the index case had a double trouble.

Since LVHT is often complicated by stroke/embolism, ventricular arrhythmias, heart failure, or sudden cardiac death, the described patient might have died from one of these intricacies.

\section{References}

1. Alston CL, Ceccatelli Berti C, Blakely EL, Oláhová M, He L, et al. (2015) A recessive homozygous p.Asp92Gly SDHD mutation causes prenatal cardiomyopathy and a severe mitochondrial complex II deficiency. Hum Genet 134(8): 869-79.

2. Towbin JA, Lorts A, Jefferies JL (2015) Left ventricular non-compaction cardiomyopathy. Lancet 336(9995): 813-825.

3. Stöllberger C, Wegner C, Finsterer J (2015) Fetal Ventricular Hypertrabeculation/Noncompaction: Clinical Presentation, Genetics, Associated Cardiac and Extracardiac Abnormalities and Outcome. Pediatr Cardiol 36(7): 1319-1326. 
4. Finsterer J, Stöllberger C, Grassberger M, Gerger D (2013) Noncompaction in mitochondrial myopathy: visible on microscopy but absent on macroscopic inspection. Cardiology 125(3): 146-149.

5. Finsterer J, Zarrouk-Mahjoub S (2015) Considerations about the genetics of left ventricular hypertrabeculation/noncompaction. Cardiol Young 25(7): 1435-1437.
6. Finsterer J (2009) Cardiogenetics, neurogenetics, and pathogenetics of left ventricular hypertrabeculation/noncompaction. Pediatr Cardiol 30: 659-681.

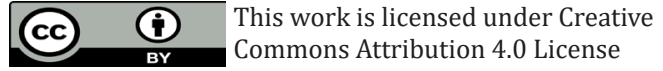

To Submit Your Article Click Here: Submit Article

DOI: $10.32474 /$ LOJMS.2018.02.000137

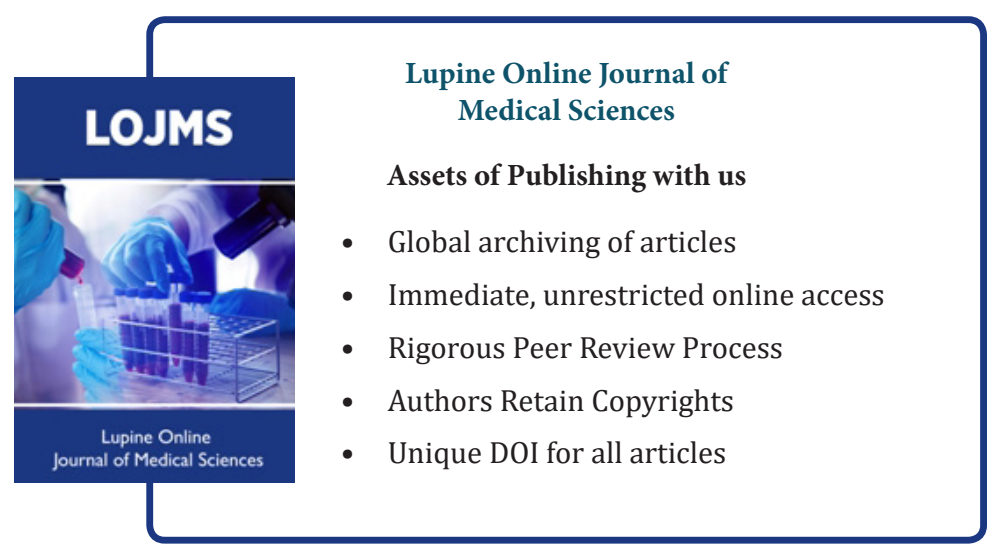

